Article

\title{
On the Application of Actively and Passively Excited Guided Elastic Waves for the Monitoring of Fiber-Reinforced Plastics ${ }^{\dagger}$
}

\author{
Kilian Tschöke*(D), Tobias Gaul ${ }^{\circ}$, Alexander Pietzsch, Eberhard Schulze and Lars Schubert \\ Fraunhofer Institute for Ceramic Technologies and Systems IKTS, 01109 Dresden, Germany; \\ tobias.gaul@ikts.fraunhofer.de (T.G.); alexander.pietzsch@ikts.fraunhofer.de (A.P.); \\ eberhard.schulze@ikts.fraunhofer.de (E.S.); lars.schubert@ikts.fraunhofer.de (L.S.) \\ * Correspondence: kilian.tschoeke@ikts.fraunhofer.de; Tel.: +49-351-88815-575 \\ t This paper is an extended version of our paper published in 12th International Workshop on Structural Health \\ Monitoring (IWSHM 2019).
}

Received: 31 March 2020; Accepted: 27 April 2020; Published: 30 April 2020

check for updates

\begin{abstract}
Nowadays, fiber-reinforced plastics are found in numerous industrial applications, such as aerospace, automotive engineering, railway, and naval engineering. These materials have high tensile and flexural strengths and nevertheless a low density at the same time. The use of fiber-reinforced plastics is particularly relevant in areas where large masses have to be moved and accelerated. However, testing and monitoring these structures is still a challenge caused by the different damage behavior compared to metal structures. Non-visible structural changes, such as delaminations and fiber-fractures, may cause local degradation and finally the failure of the components. In this work, active and passive ultrasonic methods based on guided elastic waves are investigated for their applicability to carbon fiber-reinforced structures. Therefore, tensile tests with cyclically increasing load are carried out on specimens with different fiber orientations until complete failure. The acoustic emissions in the specimen during the load are recorded. As a second technique, actively excited guided waves are transmitted and received during the rest periods between the measuring ramps. Different parameters are extracted from the measured data, which allow the monitoring of the specimen's degradation. A comparison of the results of the active and passive method follows. Finally, a combination of both methods is carried out addressing issues like its informative value and its sensitivity.
\end{abstract}

Keywords: structural health monitoring; guided waves; acousto ultrasonic; acoustic emission testing; hybrid method; fiber-reinforced composites

\section{Introduction}

Evaluating the structural state of fiber reinforced composites is still a challenge, e.g., in aerospace and automotive industry. Composite materials have a more complex damage behavior than isotropic materials. Therefore, fiber reinforced structures are very often overdesigned to tolerate damages of a certain shape and size. The full potential of such structures can not be utilized and the total weight of a component increases.

Different non-destructive testing (NDT) methods exist for the evaluation of damages today. Volume-testing methods such as computer tomography and ultrasonic techniques are most widely used. Using X-ray is complicated by radiation safety regulations and access requirements. Therefore, it is used primarily for component testing and laboratory applications. Ultrasound techniques like Pulse-Echo and Phased Array are used in aerospace for periodic testing. Again, the component has 
to be well accessible and testing becomes more and more complicated with increasing curvature of the component.

Structural Health Monitoring (SHM) methods based on guided elastic waves are an alternative to the previously described techniques. SHM is a continuous or periodic automated method for the determination and monitoring of the state of the object under surveillance within the condition monitoring (according to ISO 17359 in Germany) [1]. This is carried out by measurements of permanently installed, e.g., integrated sensors, and by the analysis of the measurement data. Therefore, the techniques differ from classic NDT measurements.

Compared to classical ultrasonic waves, guided waves propagate at lower frequencies and are characterized by more complex wave motions. They can be divided into passively and actively excited waves. For non-destructive testing and the evaluation of the structural state of a component, excited acoustic emissions can be evaluated regarding their source mechanisms and the damage mechanisms leading to these emissions. In particular, identifying damage mechanisms is challenging since a lot of damage may cause acoustic emissions at the same time. A received signal is usually the sum of acoustic emissions of different damages, which makes an interpretation more difficult. For the application of actively excited guided waves, signal features of transmitted wave packages between several transducers are evaluated to identify structural changes. Therefore, the arrangement of the transducers, the choice of an appropriate wave mode as well as the frequency are important to get an interaction with damage.

The present work is structured as follows. In Section 2, the basics of monitoring with the help of acoustic emission as well as actively excited guided waves are explained. Section 3 describes the structure and the procedure of the performed experiments. Specific damages in carbon fiber-reinforced specimen are generated during coupon testing. Afterwards, the results of the acoustic emission measurements are shown. This is followed by the results of the measurements with actively excited guided waves and a combined evaluation of the two measuring methods. A comparison of the results of simultaneously measured actively and passively excited waves is as follows. Section 4 summarizes the content and the findings of the present work.

\section{State of the Art and Methodology}

\subsection{Acoustic Emission (AE)}

Technical structures are under high loads, which are caused by aging, mechanical, or thermal stresses and lead to microscopical or macroscopical changes in the material. If these stresses exceed a threshold, energy will be discharged as an elastic wave. Typical sources of acoustic emission are friction noise, crack growth, and plastic deformation. Depending on the formation mechanism of the acoustic emission, the frequency range is typically between $1 \mathrm{kHz}$ and $1 \mathrm{MHz}$. A well-known application of acoustic emission is the monitoring of flat tank bottoms for corrosion damage and leakage [2]. The acoustic emission method is also capable of detecting the beginning and growth of cracks. Measurements during creep tests at multiple pipe sections show the applicability of acoustic emission at temperatures up to $600^{\circ} \mathrm{C}[1]$.

Already in the 1950s, Joseph Kaiser investigated the acoustic behavior of metals under stress. The Kaiser effect describes the absence of acoustic emissions until the maximum load previously reached has been exceeded [3]. Since that time, many researchers have been studying the occurrence of acoustic emissions in different materials [4-6].

In recent years, there have been an increasing number of studies dealing with carbon fiber-reinforced plastic (CFRP), which is also part of this work. Besides the Kaiser effect, another characteristic occurs on CFRP, the so-called Felicity effect. This effect refers to the fact that the pre-damage of a structure affects the acoustic emission activity of following loads. Therefore, acoustic emission can also occur during cycling loads even before reaching the previous load [7]. 
One of the first approaches to describe different damage mechanisms on fiber-reinforced plastics was described by Bohse [8]. He investigated glass fiber-reinforced materials using the double cantilever beam test. Thus, delaminations were generated in the material whose occurrence was simultaneously detected by AE sensors. He identified specific frequency ranges by a combination of source localization and frequency analysis. Emissions caused by matrix failure were indicated between 100 and $350 \mathrm{kHz}$ and caused by fiber failure between 350 and $700 \mathrm{kHz}$, respectively.

In his dissertation, Sause investigated the identification of different damage mechanisms using pattern recognition and several other analysis methods $[9,10]$. Primarily, he investigated coated CFRP specimens. The evaluation of the acoustic emissions was based on amplitude as well as energy analysis and clustering of these data. In further investigations, Sause simulated the propagation of AE signals and used the obtained data for cluster analysis. Then, he compared these results with experimental data from tensile and bending tests. He used combinations of different parameters in the frequency domain such as the Weighted Peak Frequency (WPF) and the Partial Power. The WPF is calculated according to

$$
f_{\mathrm{WPF}}=\sqrt{f_{\text {peak }} \cdot f_{\text {centroid }}}
$$

using the frequency centroid

$$
f_{\text {centroid }}=\frac{\int f \cdot \hat{U}(f) \mathrm{d} f}{\int \hat{U}(f) \mathrm{d} f}
$$

where $f_{\text {peak }}$ is the frequency of maximum signal contribution and $\hat{U}(f)$ describes the Fourier transform of the signal $U$. Sause introduced the Weighted Peak Frequency to combine the efficiency of the peak frequency with the information about the average frequency [3]. Furthermore, Sause defined

$$
\text { Partial Power } n=\frac{\int_{f_{1}}^{f_{2}} \hat{U}^{2}(f) \mathrm{d} f}{\int_{0}^{f_{\max }} \hat{U}^{2}(f) \mathrm{d} f},
$$

where $\left[f_{1}, f_{2}\right]$ is the frequency range of interest and $\left[f_{0}, f_{\max }\right]$ is the frequency range of investigation. The Partial Power 1 is determined between $f_{1}=0 \mathrm{kHz}$ and $f_{2}=150 \mathrm{kHz}$ and the Partial Power 2 between $f_{1}=150 \mathrm{kHz}$ and $f_{2}=350 \mathrm{kHz}$, respectively. Finally, Sause compared the results of simulation and experiments with microscopic investigations. By clustering the data, he succeeded in classifying the acoustic events into matrix, interface, and fiber failure based on their frequency characteristics. Nevertheless, particular clusters can not be separated from each other depending on the material, the design of the specimen, and the distance of the AE sensors. This overlapping must be taken into account in the evaluation.

\subsection{Active Guided Waves}

Guided elastic waves propagate between the surfaces of plate-like structures and are propagable over long distances. By superposition of longitudinal and transversal waves, different modes can be excited. Three different types of modes can be differed: antisymmetric modes $(A n)$, symmetric modes $(S n)$, and shear horizontal modes $(\mathrm{SHn})$, respectively. Depending on the direction of excitation, structure, thickness, and material properties as well as on the frequency, these modes are stimulated in different ratios. The velocity of the modes depends not only on the material but also on the frequency. Phase velocity, which is the propagation velocity of a specific frequency, as well as group velocity, which is the propagation velocity of a transient wave package, can be read from dispersion diagrams [11]. Depending on the frequency, different displacement mode shapes exist. This mode 
shape is also important for the interaction of the wave with defects. Depending on the type of defect, different modes and frequencies are interacting with the defect. Three different kinds of interaction can be used for guided wave-based methods, which are similar to those used for Pulse-Echo ultrasonic measurements and are the change of transmission, additional reflection, and mode conversion [12].

Using guided waves is influenced by several parameters. These are the elastic properties of the material as well as environmental and operational conditions such as load changes, temperature changes, and moisture. For isotropic materials, guided waves have been investigated for more than 30 years [11,13]. In addition, for anisotropic materials, using guided waves becomes more and more popular due to their capability to detect delaminations, which is often not possible by visual inspection [14-16].

Investigations on unidirectional reinforced specimen by Tan show the potential of guided waves in detecting delaminations [17]. Delaminations are caused, for example, by impact damage in composites $[14,18,19]$. In contrast, Tan has used different tapes to generate artificial delaminations. Furthermore, Tan applied angle probes to excite and to receive mainly the $S 0$ mode. The results showed a significant drop in the transmitted wave energy when the receiver was positioned above or behind the delamination. By this effect, it was also possible to determine the size of a delamination. Compared to conventional ultrasonic testing, even a higher accuracy was achieved when the damage was close to the surface.

With a distributed network of piezoelectric transducers that excite guided waves in a narrow-band frequency range, Su verified the existence and the position of artificial delaminations [20]. He used a quasi-isotropic plate made of CFRP with a thickness of $1.3 \mathrm{~mm}$. By using a wavelet transformation, $\mathrm{Su}$ was able to suppress noise and to identify the first arrived $S O$ mode. With the help of a straightforward location routine, the position of the damage could be estimated.

The studies mentioned above showed the potential to detect damages, such as delaminations and cracks, in fiber-reinforced composites. However, these studies contain mainly artificial defects. In contrast, composite structures are also subject to strong bending and tensile loads, depending on the field of application (aerospace, automotive engineering, railway, naval engineering). These loads may cause matrix and fiber failures. By Schubert, it has been shown that tensile tests stimulate matrix cracking, fiber matrix debonding and fiber crack, whereas bending tests lead to matrix cracking, fiber matrix debonding, and delaminations [21]. In summary, only a few papers deal with the investigation on real damaged specimen and use dynamic load tests to damage fiber-reinforced structures. Therefore, the test specimen within the presented work are loaded in a tensile test step by step up to the maximum load, generating fiber-matrix debonding and fiber cracks.

\section{Results}

\subsection{Experimental Setup}

For the investigations presented within this paper, specific specimens were loaded in tensile tests until mechanical rupture. For the tests, the testing machine "AllroundLine Zwick 100" by Zwick and Roell with hydraulic clamping jaws was used. The test regime was a stepwise tensile test, in which the specimens were cyclically loaded and unloaded. The progress of the load applied can be seen in Figure 3. With each load level, the maximum load of the previous level was increased until reaching the failure load. As an initial state, a load level of $50 \mathrm{~N}$ was applied to the testing machine. To be more precise, these $50 \mathrm{~N}$ initially applied correspond to a preload and are not part of the loading and unloading cycle. The load was increased stepwise by $1000 \mathrm{~N}$ until the specimen completely failed. During the loading of the specimen, acoustic emissions were recorded, whereas guided wave measurement data were collected during the intermission of the load steps while the preload was applied (Figure 1). Since the phase velocity of guided waves is influenced by a pre-stressing within the material, this pre-stressing should be kept constant for each measurement. 
Within this paper, the test specimens are with eccentric notches as shown in Figure 1 . The used tensile test specimens had a length of $250 \mathrm{~mm}$, a width of $25 \mathrm{~mm}$ and a thickness of $2 \mathrm{~mm}$. A total of 10 specimens with two different layer stackups were used. One layer stackup is a symmetric $0^{\circ} / 90^{\circ}$ composite, while the other stackup is a symmetric $+45^{\circ} /-45^{\circ}$ configuration. For measurements with actively excited guided waves, two "DuraAct P-876.K025" by PI Ceramic were used, which were integrated into the composite during manufacturing. Passive measurements were carried out using four "KAR601" acoustic emission sensors attached at the clamping jaws of the testing machine. To improve the measurement conditions, an ultrasonic coupling gel was used as couplant between the AE sensors and the jaws and the sensors were fixed by a yoke. The measurement system was the MAS 2 (Multi Channel Acoustic Measurement System) developed at Fraunhofer IKTS. As a big advantage, active and passive measurement data can be collected with only one hardware platform.
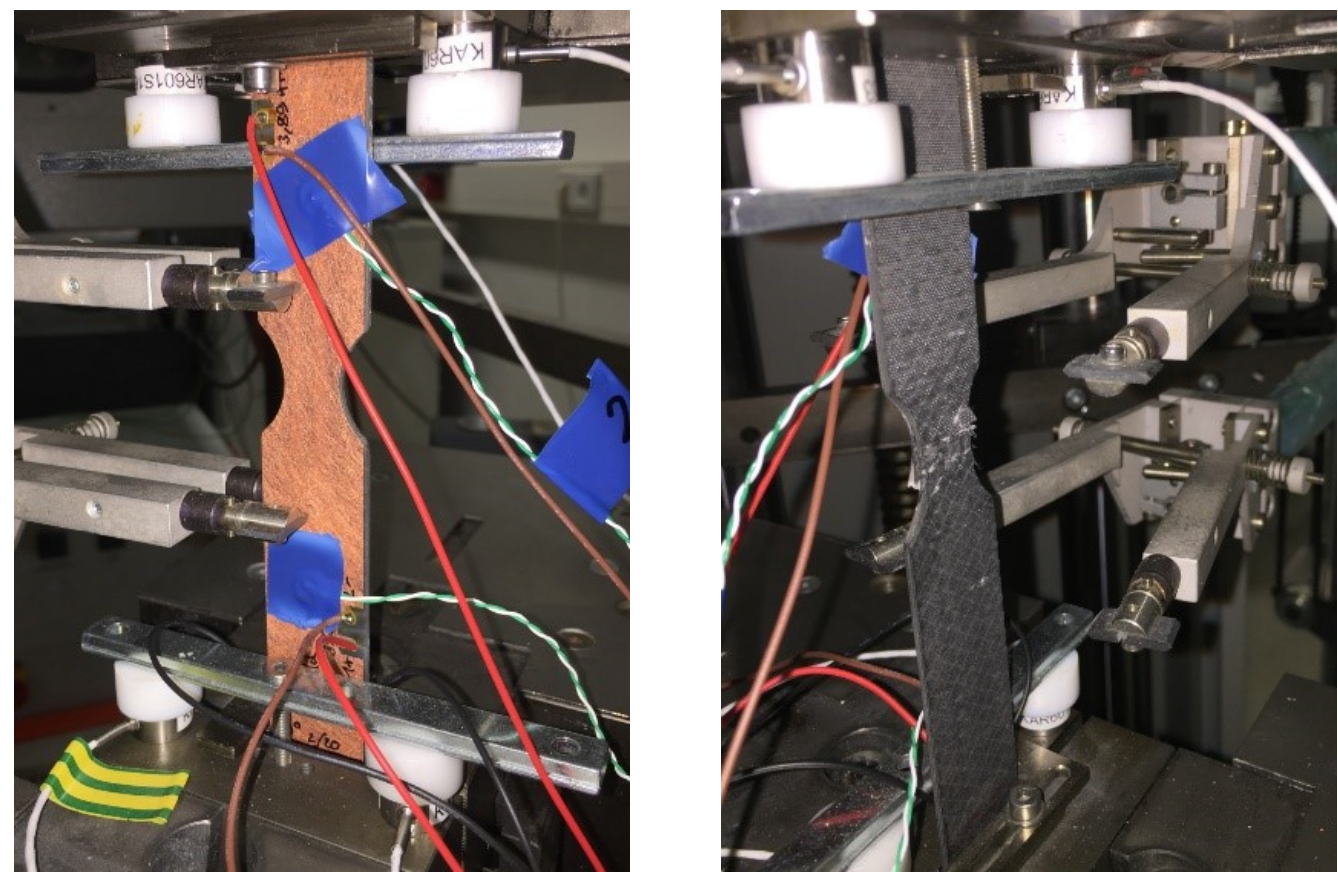

Figure 1. Clamped test specimen with embedded transducers for guided wave measurements and attached sensors for acoustic emission measurements. Left: Test specimen before starting the measurement. Right: Test specimen after successful test.

\subsection{AE Results}

During the load cycles, acoustic emissions were recorded on all specimens. In a first step, the Weighted Peak Frequency as well as the Partial Power according to Equation (1) were calculated in several frequency bands for all acoustic events. Figure 2 shows the WPF and the Partial Power 2 for all test specimens. Due to different damage mechanisms in CFRP, three different clusters can be identified according to presented results by Hönig [21]. The first cluster is characterized by acoustic emission events with a mean WPF of around $150 \mathrm{kHz}$ and a Partial Power between $1 \%$ and $60 \%$. These events can be assigned to matrix failures within the material. In the second cluster, acoustic emission events accumulate mainly around $195 \mathrm{kHz}$ with a Partial Power between 5\% and 45\%. These results are related to fiber matrix debonding. A third cluster is formed with a mean WPF of $250 \mathrm{kHz}$ with Partial Powers between $60 \%$ and $95 \%$. This cluster is explained by fiber cracks. In the further work, these clusters will be used to assign corresponding damage mechanisms to occuring AE signals.

Figure 3 shows an example of the test sequence of a 0/90 specimen. The WPF (red dots) as well as the location of the acoustic events (blue crosses) are presented during the measurement time. At the beginning of the load cycles, only a few AE events with low WPF occur. These events are distributed irregularly and close to the integrated "DuraAct" transducers. With increasing load, the number of 
acoustic events also increases significantly. The locations of these events are focused in the area of the notch of the specimen where the failures would be expected. In addition to numerous events with a WPF around $150 \mathrm{kHz}$ caused by matrix cracks, events with a WPF around $200 \mathrm{kHz}$ and above occur. The last-mentioned events are caused by fiber matrix debonding and fiber cracks and indicate a failure of the specimen.

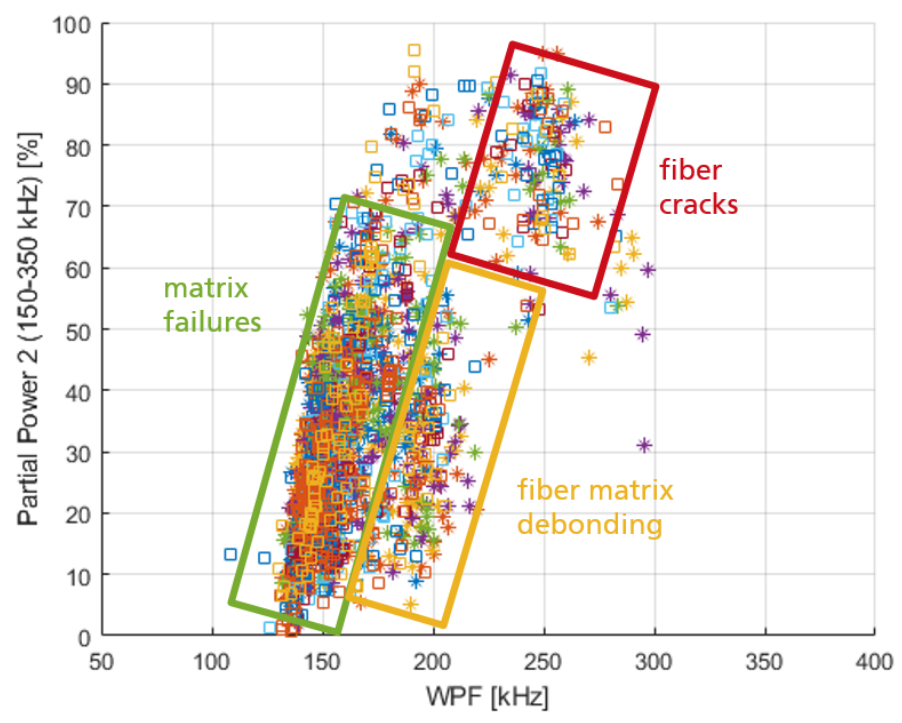

Figure 2. Partial Power 2 vs. Weighted Peak Frequency for all test specimens. Additionally, the clusters of damage mechanisms are shown. Asterisks: 0/90 specimens. Squares: $+45 /-45$ specimens.

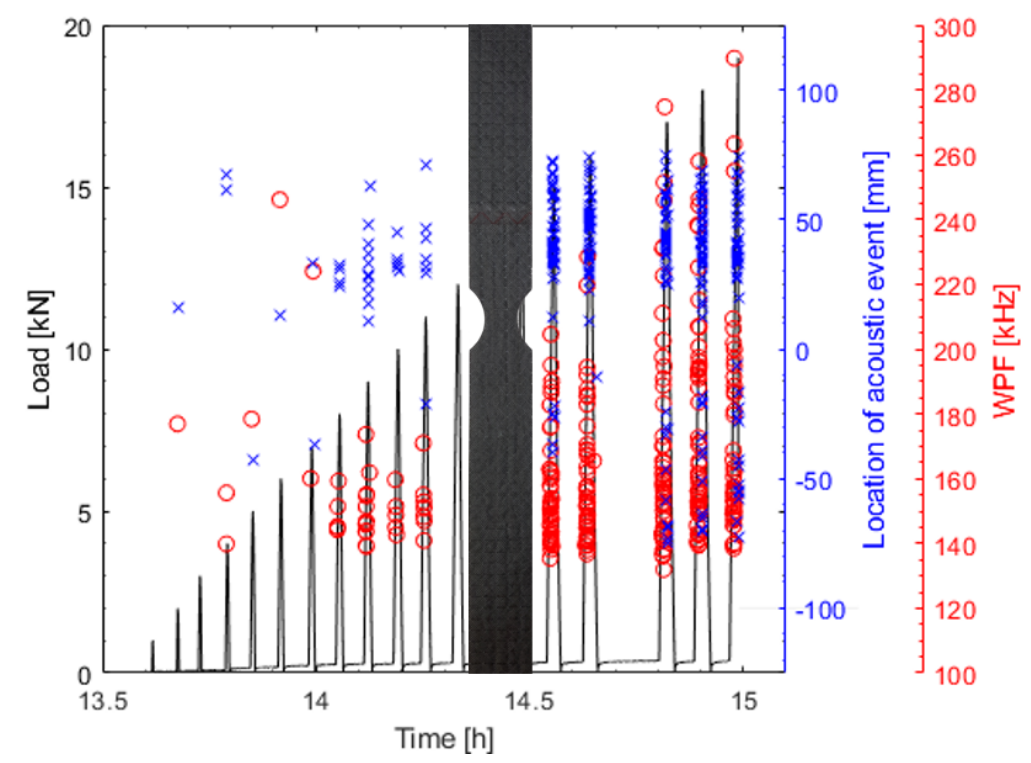

Figure 3. Principle behavior of the specimens during the tensile test. During the beginning of the test, matrix failures occur close to the sensor locations (red dots at lower levels of the WPF). At the end of the test, fiber cracks as well as debonding occur in the area of the notch of the specimen (red dots at high levels of the WPF).

\subsection{Results of Actively Excited Guided Waves}

The results of the AE measurements show clearly the damage progression of the tested specimen with increasingly applied load. However, compared to AE measurements, less data are available with actively excited guided waves. For each load level, only one dataset with new information can be 
extracted since the data were collected during the intermission of the load steps. So-called damage indices are determined from the time signals.

Figure 4 shows examples of the time signals vs. the load levels of two test specimens. The dominant wave modes are around $45 \mu$ s (left) and $50 \mu$ s (right). This wave mode has a significant reduction of the amplitude as well as an increasing phase shift from a load level of $10 \mathrm{kN}$ for the $0 / 90$ specimens and of $7 \mathrm{kN}$ for the $+45 /-45$ specimens. From this load level, an increased amount of acoustic events was also realized (Figure 3). Due to the amount of acoustic events and the corresponding damage mechanism presented in Section 3.2, it can be concluded that the specimen was strongly damaged during the experiment. It is now to be investigated whether changes in the test specimen can also be detected with the help of the actively excited guided wave measurements, in particular with measurements in the above-mentioned load levels.
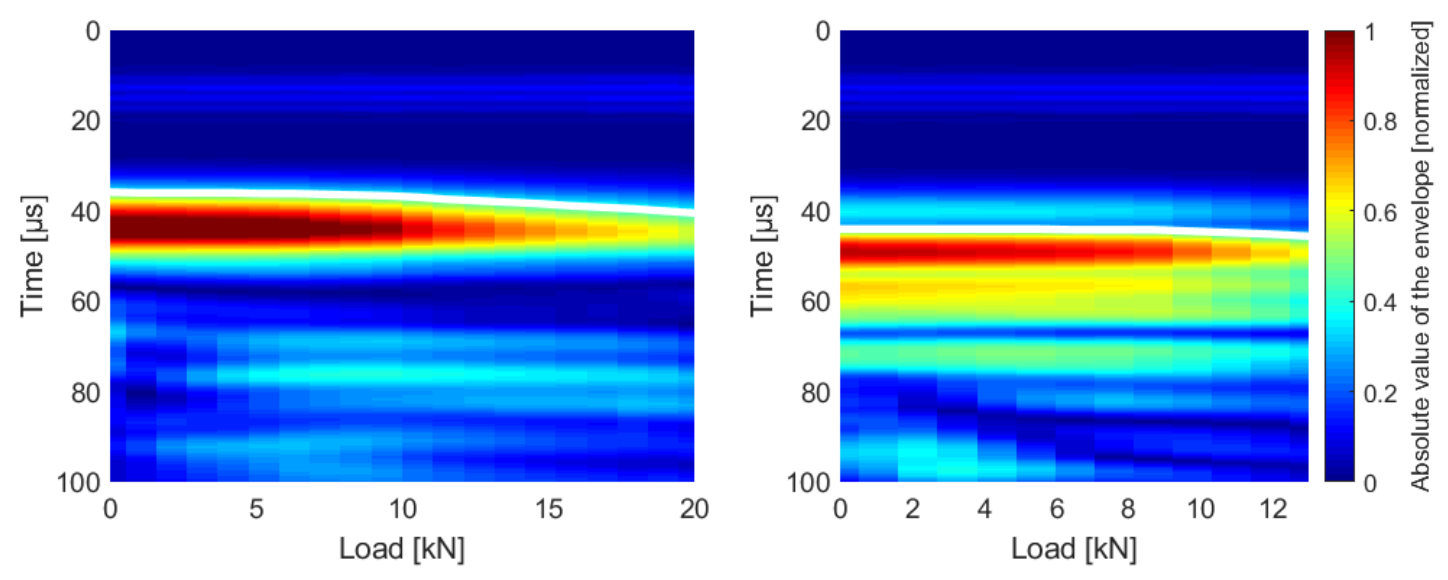

Figure 4. Absolute value of the envelope of measured time signals after each load level for a $0 / 90$ and a $+45 /-45$ test specimen. The white line indicates the arrival time of the wave package at the sensor. Left: $0 / 90$ test specimen $1-20$. Right: $+45 /-45$ test specimen $1-10$.

The dominant wave mode is extracted from the time signal and used to calculate damage indices $D I$. The DIs describe the change in the time signal, and hence the specimen condition. The change in the energy of the mode is calculated using the difference of the mean square value between the measured time signal $x=\left\{x_{i}\right\}_{i=1}^{M}$ of a reference state and the measured time signal $y=\left\{y_{i}\right\}_{i=1}^{M}$ of a load state according to Park [22]:

$$
D I_{\text {rmsd }}=100 \cdot \sqrt{\frac{\sum_{i=1}^{M}\left(y_{i}-x_{i}\right)^{2}}{\sum_{i=1}^{M} x_{i}^{2}}} .
$$

The change in the phase of the mode is determined by the correlation coefficient from the covariance matrix $V$ of both signals $\left\{x_{i}\right\}_{i=1}^{M}$ and $\left\{y_{i}\right\}_{i=1}^{M}$ according to Mueller [23]:

$$
D I_{C C}=1-\left|\frac{V_{12}}{\sqrt{V_{11} \cdot V_{22}}}\right| .
$$

Here, $V$ is defined by

$$
V=\left(\begin{array}{ll}
V_{11} & V_{12} \\
V_{21} & V_{22}
\end{array}\right)=\left(\begin{array}{ll}
\operatorname{cov}(x, x) & \operatorname{cov}(x, y) \\
\operatorname{cov}(y, x) & \operatorname{cov}(y, y)
\end{array}\right),
$$


where

$$
\operatorname{cov}(x, y)=\frac{1}{M-1} \sum_{i=1}^{M}\left(x_{i}-\mu_{x}\right) \cdot\left(y_{i}-\mu_{y}\right)
$$

with

$$
\mu_{x}=\frac{1}{M} \sum_{i=1}^{M} x_{i} \quad \text { and } \quad \mu_{y}=\frac{1}{M} \sum_{i=1}^{M} y_{i} .
$$

Figure 5 shows the change in the energy of the wave mode for all test specimens, the corresponding mean values and the standard deviation, respectively. Comparable trends of the damage index are shown for the $0 / 90$ as well as for the $+45 /-45$ test specimens. The $0 / 90$ specimens have a small amplitude decrease up to $10 \mathrm{kN}$ and a steeper, but steady, negative slope from $10 \mathrm{kN}$. Since only one $0 / 90$ specimen has reached a load level of more than $20 \mathrm{kN}$, no standard deviation can be determined from $20 \mathrm{kN}$. For the $+45 /-45$ specimens, the turning-point of the damage index occurs at $7 \mathrm{kN}$ and the damage index shows a negative slope up to the failure threshold at $14 \mathrm{kN}$.
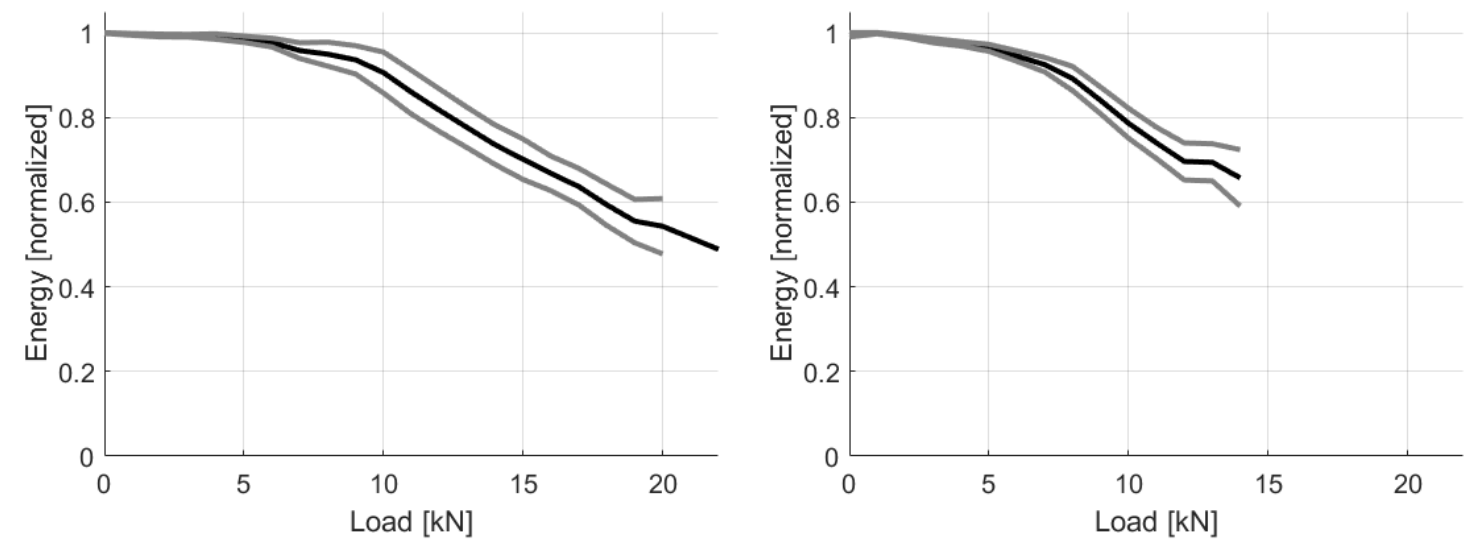

Figure 5. Mean value (black) and standard deviation (gray) of the energy of the evaluated wave package of all test specimens. Left: $0 / 90$ test specimens. Right: $+45 /-45$ test specimens.

For both kinds of test specimens, three intervals are visible. The first interval at low load levels is characterized by minor changes of the damage index. It can be assumed that the stretching of the test specimen may change the initial state at the load level of $50 \mathrm{~N}$ compared to the reference state. This change is indicated by the damage index. In a second interval, there is a larger change of the $D I_{r m s d}$, which may indicate beginning damages such as inter-fiber cracks. With increasing load levels, there is a turning-point in the curve and the change in the $D I_{r m s d}$ is almost linear until the specimen completely fails. In addition to numerous inter-fiber cracks, fiber cracks in the area of the notch also occur (Figure 3).

\subsection{Combination of Results}

The results presented above show the results of $\mathrm{AE}$ and guided wave measurements separately and will be now combined for joint interpretation. In particular, due to the monotonic change of the damage index of the guided wave measurements, the DI can be used for weighting the AE events. Figure 6 shows an example of such a combination of results for two different test specimens. The figure presents the number of recorded acoustic events color-coded in a given frequency band with a width of $12 \mathrm{kHz}$ and the curve of the damage index $D I_{C C}$. One can see a significant increase in the damage index from $12 \mathrm{kN}$ for the $0 / 90$ specimens and from $10 \mathrm{kN}$ for the $+45 /-45$ specimens, respectively. Both slopes correlate with an increase of acoustic events with frequencies between 144 and $156 \mathrm{kHz}$ 
from the mentioned load level. Until the specimen is destroyed, damage index and acoustic events continue to increase.
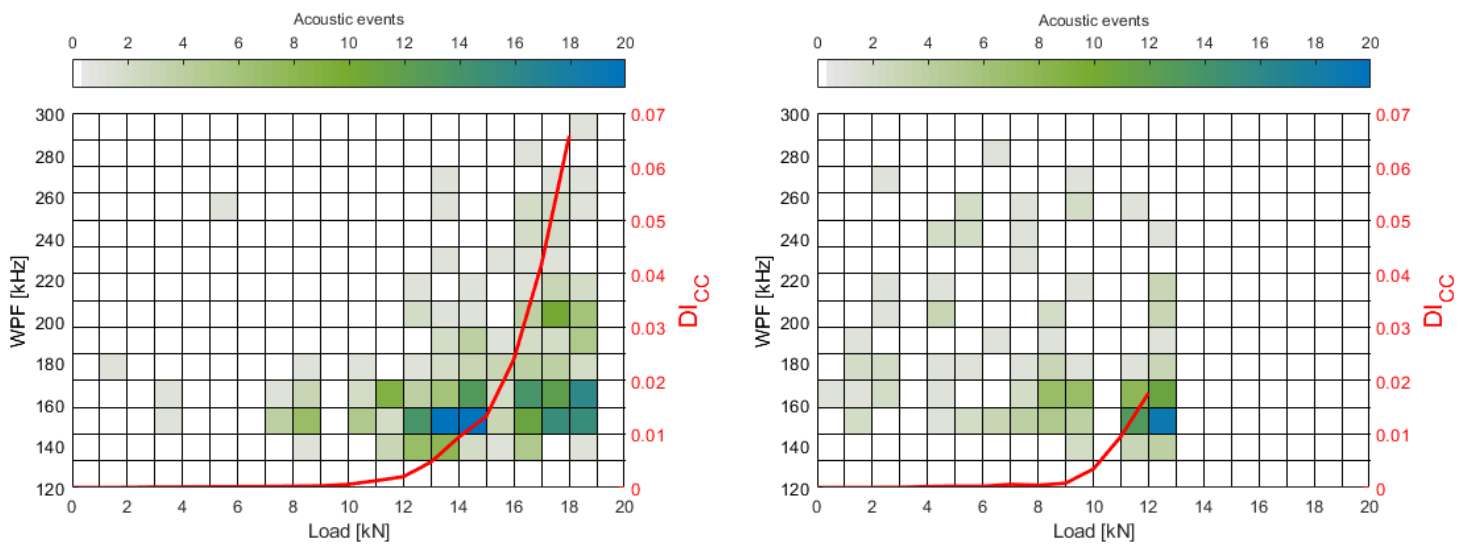

Figure 6. Combination of AE and guided wave measurements. The damage index $D I_{C C}$ (red line) and the recorded acoustic events (color-coded) vs. the load levels and the WPF are plotted. Left: 0/90 test specimen 2-20. Right: $+45 /-45$ test specimen 1-10.

Alternatively, Figure 7 combines the results of AE and guided wave measurements by representing the mean value and the standard deviation of the energy of the evaluated wave package for all 0/90 and for all $+45 /-45$ test specimens, respectively. The cumulative percentage of the WPF in a specific frequency band for each load level is color-coded. The acoustic events significantly increase from a load level of $13 \mathrm{kN}$ for the $0 / 90$ test specimens and from $9 \mathrm{kN}$ for the $+45 /-45$ specimens. These events have a WPF around $150 \mathrm{kHz}$ and indicate matrix failures. From these load levels, the specimens begin to suffer damages which finally lead to failure. From a load level of $10 \mathrm{kN}$ for the $0 / 90$ test specimens and from $7 \mathrm{kN}$ for the $+45 /-45$ specimens, the trends of the energy curves show an explicit change. One can conclude that a change is taking place in the test specimens. The knowledge from the acoustic emission measurements allow a statement about the damage occurred. Hence, the trend of the damage index $D I_{r m s d}$, which represents the energy of the evaluated wave package, shows a significant change as soon as matrix failure occurs. By combining the results, it can be concluded that this damage index can also be used to identify relevant damage mechanisms.
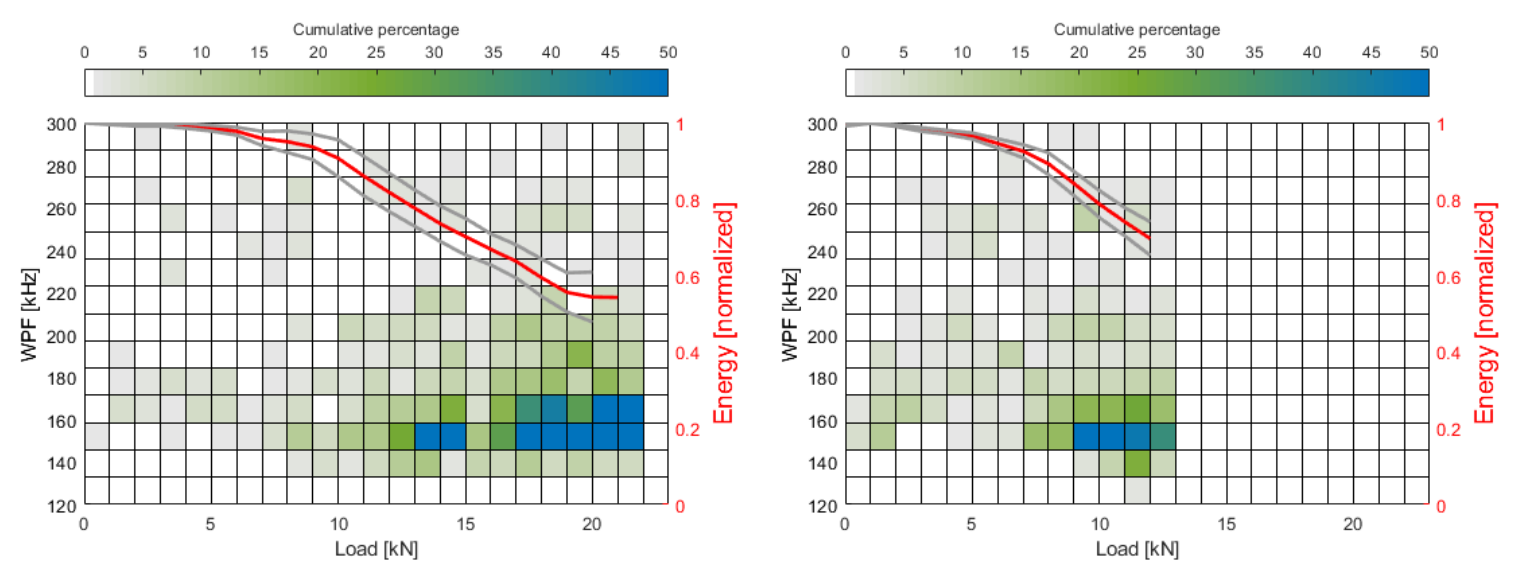

Figure 7. Mean value (red) and standard deviation (gray) of the energy of the evaluated wave package. Color-coded is the cumulative percentage of the WPF in a frequency band for each load level. Left: 0/90 test specimen $2-20$. Right: $+45 /-45$ test specimen $1-10$.

Figure 8 shows the locations of the acoustic events and the damage index $D I_{C C}$ for two selected test specimens. For the $0 / 90$ specimen, it can be seen that the acoustic events are localized throughout the 
specimen for $D I_{C C}$ values below 0.005 . When the damage index exceeds a value of 0.005 , the acoustic events focus in the area of the notch. Since the failure of the specimen is expected in this area, one can conclude that a beginning failure of the specimen can be detected from this value of the $D I_{C C}$. An equivalent behavior can be seen for the $+45 /-45$ test specimen. Hence, the value of the damage index, from which the acoustic events are focused in the area of the notch, is around 0.0025 and lower compared to the $0 / 90$ specimen. Thus, one can define a threshold for the damage index, which indicates a damage of the specimen, and one can reduce the amount of AE data without losing informative values to determine the damage.
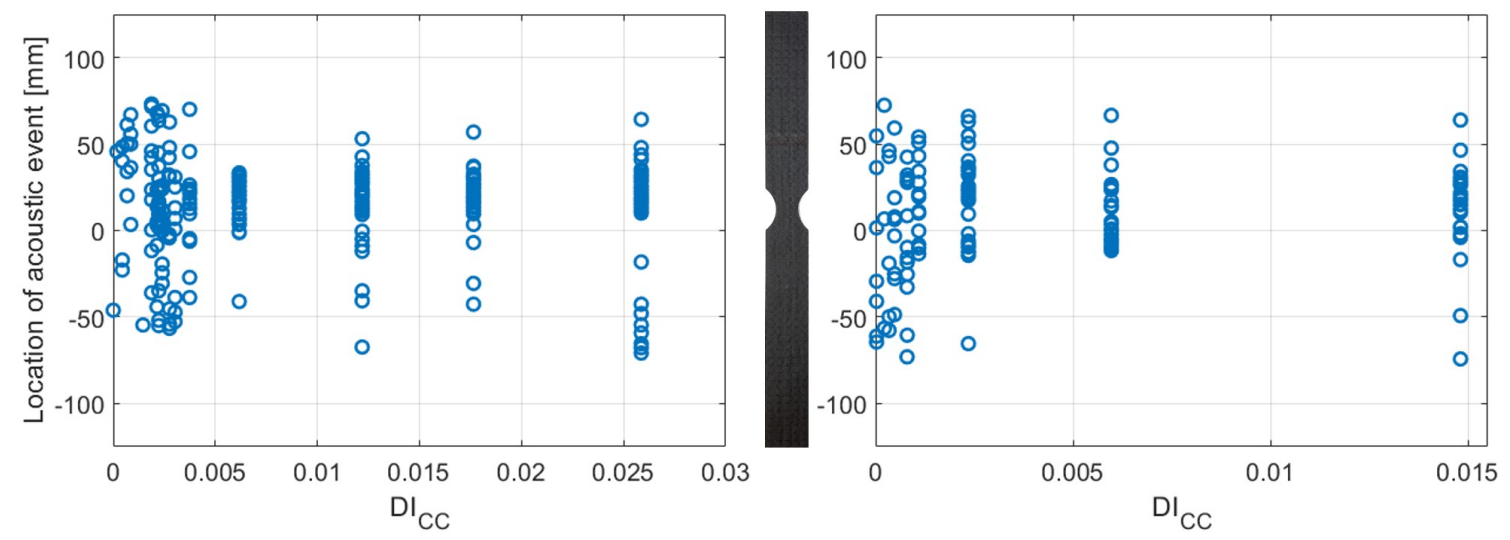

Figure 8. Location of the acoustic events vs. damage index $D I_{C C}$. Left: $0 / 90$ test specimen. Right: $+45 /-45$ test specimen.

Figure 9 shows the Partial Power 2 and the WPF of the acoustic emission events for all test specimens. The chart on the left side corresponds to Figure 2 and the chart on the right side shows the result after reducing the number of AE events using the threshold of the damage index. The amount of AE data could be reduced by about $30-40 \%$. Nevertheless, the clusters of damage mechanisms are still clearly recognizable.
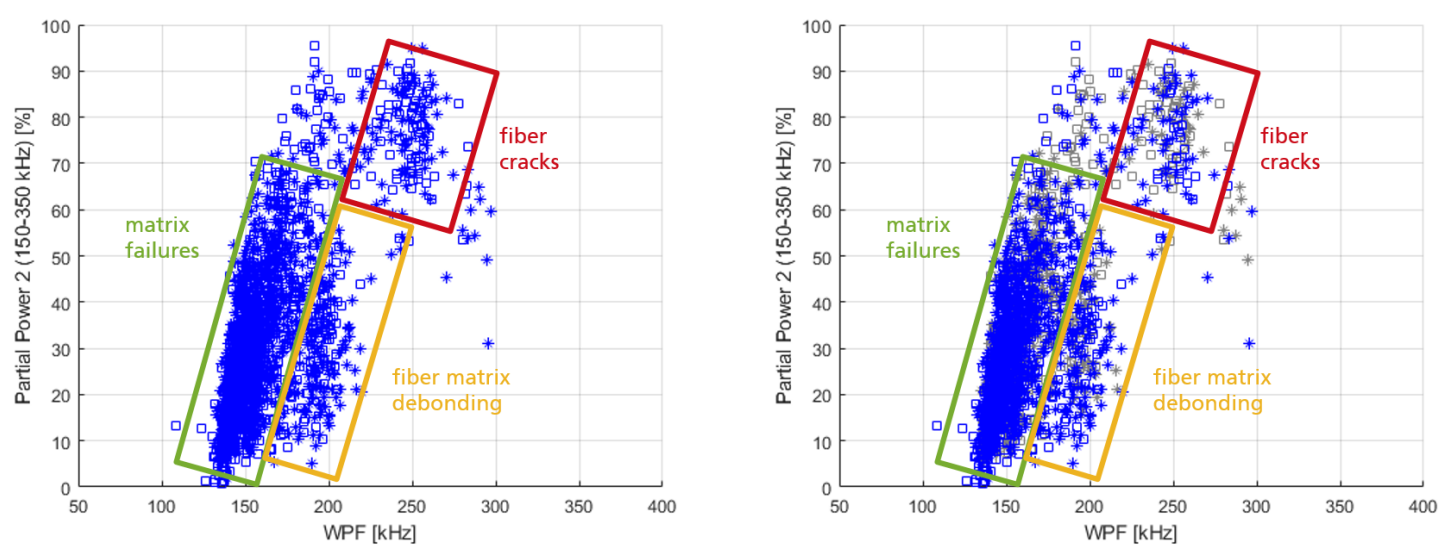

Figure 9. Partial Power 2 vs. WPF for all test specimens. Left: All acoustic events are regarded (Figure 2). Right: Acoustic events after considering a threshold of the damage index based on Figure 8. The hidden AE events are highlighted in gray, those still considered in blue. One can see a reduction of the data amount of around $30-40 \%$.

\section{Conclusions}

The present work shows the potential to detect damages in CFRP by a combination of actively and passively excited guided elastic waves. It could be shown that there is a relationship between the occurrence and intensity of acoustic emission events and the rate of change of a calculated damage 
index on a path between two transducers. Further work will investigate how to extend the results on a laboratory scale to real structures. Especially for large structures, it is necessary to place a sensor network on or in the component to ensure a large number of travel paths for the evaluation of a damage index.

Furthermore, the calculated damage index can be used to significantly reduce the amount of data without losing informative values. In particular, this seems to be useful for long-term monitoring, where a large amount of data are generated and a user must decide on the basis of the AE events whether damages of a significant size have occurred or not. However, it has to be investigated if this reduction of $\mathrm{AE}$ data are useful for fast-changing processes. At the same time, a threshold can be specified, from which the damage index indicates a damage process within the structure. The acoustic emission parameters Weighted Peak Frequency and Partial Power were used to identify and classify the damage.

Author Contributions: Conceptualization, T.G., L.S., and K.T.; methodology, E.S. and T.G.; software, E.S. and T.G.; validation, T.G. and E.S.; formal analysis, T.G. and E.S.; investigation, A.P.; writing-original draft preparation, T.G., L.S., and K.T.; writing-review and editing, K.T.; visualization, T.G. and K.T.; supervision, L.S.; project administration, K.T.; funding acquisition, L.S. and K.T. All authors have read and agreed to the published version of the manuscript.

Funding: This research was founded by the German Federal Ministry of Education and Research in the project "CarbonSafe" (funding code: 16ES0333). The Article Processing Charge (APC) was funded by the publication fund of the Fraunhofer-Gesellschaft.

Conflicts of Interest: The authors declare no conflict of interest. The funders had no role in the design of the study; in the collection, analyses, or interpretation of data; in the writing of the manuscript, or in the decision to publish the results.

\section{Abbreviations}

The following abbreviations are used in this manuscript:

AE Acoustic Emission

CC Correlation Coefficient

CFRP Carbon Fiber-reinforced Plastics

DI Damage Index

MAS Multi-Channel Acoustic Measurement System

NDT Non-Destructive Testing

RMSD Root-Mean-Square Deviation

SHM Structural Health Monitoring

WPF Weighted Peak Frequency

\section{References}

1. Weihnacht, B.; Lieske, U.; Gaul, T.; Tschöke, K. Handbook of Advanced Non-Destructive Evaluation; Chapter Structural Health Monitoring; Springer Nature Switzerland AG: Berlin/Heidelberg, Germany, 2018; pp. 1-19.

2. Tscheliesnig, P.; Lackner, G.; Jagenbrein, A. Corrosion detection by means of acoustic emission (AE) monitoring. In Proceedings of the 19th World Conference of Non-Destructive Testing, Munich, Germany, 13-17 June 2016.

3. Sause, M. In Situ Monitoring of Fiber-Reinforced Composites; Springer Series in Materials Science; Springer: Berlin/Heidelberg, Germany, 2016; Volume 242.

4. Ono, K. (Ed.) Advances in Acoustic Emission. In Proceedings of the Sixth International Conference on Acoustic Emission, Lake Tahoe, NV, USA, 29 October-2 November 2007; The Acoustic Emission Working Group and Acoustic Emission Group: Encino, CA, USA, 2007.

5. Grosse, C.U.; Ohtsu, M. Acoustic Emission Testing; Springer: Berlin/Heidelberg, Germany, 2008.

6. Sikhorski, W. Acoustic Emission; InTech: Rijeka, Croatia, 2012.

7. Rowland, C. Acoustic emission technique to assist the formula one designer in structural design. In Proceedings of the European Working Group on Acoustic Emission (EWGAE), Tokyo, Japan, 9-12 November 2004. 
8. Bohse, J.; Chen, J. Acoustic emission examination of Mode I, Mode II and mixed Mode I/II interlaminar fracture of unidirectional fibre reinforced polymer. J. Acoust. Emiss. 2001, 19, 1-10.

9. Sause, M.; Schultheiß, D.; Horn, S. Acoustic emission investigation of coating fracture and delamination in hybrid carbon fibre reinforced plastic structures. J. Acoust. Emiss. 2008, 26, 1-13.

10. Sause, M. Identification of Failure Mechanisms in Hybrid Materials Utilizing Pattern Recognition Techniques Applied to Acoustic Emission Signals. Ph.D. Thesis, University Augsburg, Augsburg, Germany, 2010.

11. Giurgiutiu, V. Struchtural Health Monitoring with Piezoelectric Wafer Active Sensors; Academic Press: Cambridge, MA, USA, 2008.

12. Mueller, I. Inspection of Piezoelectric Transducers Used for Structural Health Monitoring Systems. Ph.D. Thesis, Universität Siegen, Siegen, Germany, 2017.

13. Su, Z.; Ye, L. Identification of Damage Using Lamb Waves-From Fundamentals to Applications. In Lecture Notes in Applied and Computational Mechanics; Springer: Berlin/Heidelberg, Germany, 2009; Volume 48.

14. Schubert, L.; Lieske, U.; Köhler, B.; Frankenstein, B. Interaction of Lamb Waves with impact damaged CFRP's studied by Laser-Vibrometry and Acousto Ultrasonic. In Proceedings of the 18th European Conference on Fracture, Dresden, Germany, 29 August-3 September 2010.

15. Frankenstein, B.; Fischer, D.; Weihnacht, B.; Rieske, R. Lightning safe rotor blade monitoring using an optical power supply for ultrasonic techniques. In Proceedings of the 6th European Workshop on Structural Health Monitoring, Dresden, Germany, 3-6 July 2012.

16. Wölcken, P.C.; Papadopoulos, M. (Eds.) Smart Intelligent Aircraft Structures (SARISTU). In Proceedings of the Final Project Conference; Springer: Berlin/Heidelberg, Germany, 2016.

17. Tan, K.S.; Guo, N.; Wong, B.S. Experimental evaluation of delaminations in composite plates by the use of Lamb waves. Compos. Sci. Technol. 1995, 55, 77-84. [CrossRef]

18. Memmolo, V.; Ricci, F.; Maio, L.; Boffa, N.D.; Monaco, E. Model assisted probability of detection for a guided waves based SHM technique. In Proceedings of the SPIE Smart Structures and Materials + Nondestructive Evaluation and Health Monitoring, Las Vegas, NV, USA, 21-24 March 2016.

19. Gaul, T.; Tschöke, K.; Schulze, E.; Schubert, L. Development and evaluation of Structural Health Monitoring systems for fibre composites in automotive application based on elastic waves. In Proceedings of the 9th European Workshop on Structural Health Monitoring, Manchester, UK, 10-13 July 2018.

20. Su, Z.; Ye, L.; Bu, X. A damage identification technique for CF/EP composite laminates using distributed piezoelectric transducers. Compos. Struct. 2002, 57, 465-471. [CrossRef]

21. Hönig, U.; Holder, U.; Pietzsch, A.; Schulze, E.; Frankenstein, B.; Schubert, L. Definition of requirements for reference experiments to determine and evaluate various damage mechanisms in fibre composites by acoustic emission. In Proceedings of the 19th World Conference of Non-Destructive Testing, Munich, Germany, 13-17 June 2016.

22. Park, S.; Yun, C.B.; Roh, Y.; Lee, J.J. PZT-based active damage detection techniques for steel bridge components. Smart Mater. Struct. 2006, 15, 957-966. [CrossRef]

23. Mueller, I.; Fritzen, C.P. Inspection of Piezoceramic Transducers Used for Structural Health Monitoring. Materials 2017, 10, 71. [CrossRef] [PubMed]

(C) 2020 by the authors. Licensee MDPI, Basel, Switzerland. This article is an open access article distributed under the terms and conditions of the Creative Commons Attribution (CC BY) license (http://creativecommons.org/licenses/by/4.0/). 